\title{
The management of gout
}

\section{SUMMARY}

Gout is a common inflammatory arthritis that is increasing in prevalence. It is caused by the deposition of urate crystals.

Non-steroidal anti-inflammatory drugs, colchicine and corticosteroids are options for the management of acute gout. They are equally efficacious and comorbidities guide the best choice.

Allopurinol is an effective treatment for reducing concentrations of uric acid. Renal function guides the starting dose of allopurinol and the baseline serum uric acid concentration guides the maintenance dose.

Febuxostat is another xanthine oxidase inhibitor. It is clinically equivalent to allopurinol.

Uricosuric drugs, such as probenecid, increase uric acid excretion. New drugs in this class will soon become available and are likely to have a role in the treatment of patients who do not respond to other drugs.

\section{Introduction}

Gout is one of the most common inflammatory arthropathies. Studies suggest a prevalence of $1.7 \%$ in Australia and $2.7 \%$ in New Zealand, with higher rates in Maori and islander populations. 'The National Health and Nutrition Examination Survey (NHANES) in the USA and studies in New Zealand, China and the UK have shown that gout and hyperuricaemia are increasing in prevalence. ${ }^{2,3} \mathrm{~A}$ study of its prevalence in Aboriginal Australians in 1965 found an absence of gout, but in 2002, the prevalence had risen to $9.7 \%$ in men and $2.9 \%$ in women. ${ }^{4}$ The prevalence of gout in the USA in 2007-08 was $6 \%$, but the prevalence of hyperuricaemia was $21 \%$. $2,4,5$

Hyperuricaemia is defined as a serum uric acid more than $0.36 \mathrm{mmol} / \mathrm{L}$ in women and more than $0.42 \mathrm{mmol} / \mathrm{L}$ in men. About $10 \%$ of people with hyperuricaemia develop gout, but $80-90 \%$ of patients with gout are hyperuricaemic. ${ }^{4,5}$ The chance of developing gout increases with increasing serum concentrations of uric acid. Why only a minority of those with hyperuricaemia develop clinical gouty arthritis is unclear. ${ }^{2}$ At present, there is insufficient evidence to recommend treatment of asymptomatic hyperuricaemia to prevent gouty arthritis, chronic kidney disease or cardiovascular events.'

Despite the high prevalence of gout and the availability of safe and effective therapies, there remains considerable practice variation in diagnosis and management.

\section{Pathophysiology}

Gout results from a raised total body uric acid concentration with consequent deposition of crystals in joints and occasionally elsewhere. Unlike most mammals, humans lack the enzyme capable of degrading uric acid. Humans tend to have far higher urate concentrations and these are linked to a constellation of clinical conditions, most notably gout. ${ }^{6}$ There are two important factors that influence uric acid concentrations in the body. These are the amount of uric acid produced and the clearance of uric acid from the body. Approximately two-thirds is removed by renal clearance and one-third by intestinal clearance.

\section{Clinical features}

Monosodium urate crystals typically form in relatively cooler parts of the body including the metatarsophalangeal joint of the big toe, the joints of the feet, knees, elbows and hands. The crystals may also deposit in the soft tissues around joints and form tophi which can also occur on the cartilage of the ears.

Gout usually presents as a painful monoarthritis that spontaneously resolves over a few days to one to two weeks. It occurs more commonly in males after puberty, and in females after menopause. Gout is characterised by recurrent flares of severe joint inflammation, but most patients are asymptomatic between attacks.?

\section{Andrew Finch}

Rheumatology and clinical pharmacology registrar

\section{Paul Kubler}

Rheumatologist and clinical pharmacologist

Royal Brisbane and Women's Hospital

Brisbane

\section{Keywords}

allopurinol, febuxostat, gout, uric acid

Aust Prescr 2016;39:119-22 http://dx.doi.org/10.18773/ austprescr.2016.047 


\section{Diagnosis}

For a definitive diagnosis of gout, urate crystals must be demonstrated in synovial fluid or in the tophus. Synovial fluid should be analysed by polarised light microscopy. Once the definitive diagnosis has been made, repeat attacks do not require diagnostic aspiration unless there is a suspicion of joint sepsis. A normal or low serum urate does not exclude the diagnosis of acute gout, ${ }^{1}$ because the concentration may not be elevated during an acute attack.

First metatarsophalangeal joint involvement, local erythema, maximal inflammation within 24 hours and hyperuricaemia are suggestive of gouty arthritis, however a response to colchicine and the presence of tophi have a higher diagnostic usefulness.' Some imaging modalities such as ultrasound and dualenergy CT scan may be helpful if the diagnosis is uncertain. ${ }^{1,7}$

Several drugs used for treatment of comorbid conditions can alter serum urate concentrations. Losartan, atorvastatin, fenofibrate and calcium channel blockers all have weak urate-lowering properties. ${ }^{8}$ Low-dose aspirin and diuretics, particularly thiazide diuretics, increase serum urate. If possible, thiazide diuretics should not be used to treat hypertension in people with gout.

It is important not to overlook other causes of hyperuricaemia. These include renal diseases and myeloproliferative disorders.

\section{Treatment of acute attacks}

The management of acute attacks focuses on the prompt treatment of inflammation and pain with the use of anti-inflammatory drugs. Non-steroidal anti-inflammatory drugs (NSAIDs), colchicine or corticosteroids are the first-line options, with the choice of drug being influenced by patient comorbidities and concomitant drugs.

Low-dose colchicine has similar efficacy to highdose colchicine with an adverse-effect profile not significantly different from placebo. ${ }^{8}$ The Australian Medicines Handbook recommends $1 \mathrm{mg}$ as soon as possible, then 500 micrograms one hour later (maximum $1.5 \mathrm{mg}$ per course). Do not repeat the course within three days. ${ }^{9}$

Colchicine is a substrate of both cytochrome P450 3A4 and P-glycoprotein so it may interact with antineoplastic drugs, calcium channel blockers (diltiazem and verapamil), calcineurin inhibitors, digoxin, dabigatran, macrolide antibiotics and protease inhibitors. ${ }^{10}$

Short-term NSAIDs (3-5 days) are effective during an acute attack. All drugs in this class have equal efficacy.
Oral prednisolone $35 \mathrm{mg}$ daily has been shown to effectively treat the symptoms of acute gout," however 15-20 mg daily is often recommended. ${ }^{12}$ It can usually be stopped after 3-5 days.

\section{Urate-lowering therapy}

After management of an acute attack, urate-lowering therapy should be considered in those with gout and at least one of the following:

- tophi

- two or more attacks a year

- chronic kidney disease (stage 2 or worse)

- urolithiasis. ${ }^{13}$

The goals of therapy are to maintain serum uric acid concentrations below a concentration at which urate crystals can form. Preventing the formation of urate crystals reduces the likelihood of joint inflammation, but there is no clear consensus about when to start.

A target serum uric acid of less than $0.30 \mathrm{mmol} / \mathrm{L}$ is recommended when tophi are present, otherwise less than $0.36 \mathrm{mmol} / \mathrm{L}$ is sufficient. ' Urate-lowering therapy should be titrated until the target is achieved. Long-term maintenance of the target concentration is recommended. Combination therapy may be required depending on the patient's tolerance and response to therapy. Investigation and treatment of conditions that predispose to gout such as the metabolic syndrome should also be undertaken.

Healthy lifestyle advice should include maintenance of ideal body weight and avoidance of excess alcohol, sugar-sweetened drinks and other known triggers identified for the individual. ${ }^{1}$ There is little evidence to support a relationship between a larger consumption of meat and the risk of triggering an attack in those with established gout. ${ }^{14}$ Avoidance of some risk factors such as seafood should be weighed against their possible cardiovascular health benefits. ${ }^{15}$

\section{Prophylaxis}

When starting urate-lowering therapy, concomitant prophylaxis should be provided for a minimum of six months to prevent flares of gout. ${ }^{7,8}$ It is common for flares of gout to occur when starting treatment and when changing the dose. Preventing these flares is a goal of treatment. NSAIDs and low-dose colchicine are first line and low-dose prednisolone is second line. ${ }^{8}$

Colchicine is equal to NSAIDs for long-term prophylaxis, however short-term NSAIDs or oral glucocorticoids may be appropriate depending on the patient's comorbidities and drugs. A dose of 500 microgram (one tablet) of colchicine twice daily for people with normal renal function, and 500 microgram daily in those with renal impairment, may be considered. 


\section{Xanthine oxidase inhibitors}

Xanthine oxidase catalyses two relevant reactions the production of hypoxanthine from xanthine and the formation of uric acid from hypoxanthine. ${ }^{8}$ The inhibition of xanthine oxidase therefore reduces not only uric acid production but also the production of the uric acid precursor. ${ }^{8}$

\section{Allopurinol}

Allopurinol is the first-line drug for urate-lowering therapy. It is a purine analogue which competitively inhibits xanthine oxidase, reducing the production of uric acid.

In patients with normal renal function, allopurinol should be started at a dose of $100 \mathrm{mg}$ daily for the first month. Increase the daily dose by $50 \mathrm{mg}$ every 2-4 weeks until the target serum uric acid concentration is reached. Plasma urate concentrations can be measured monthly during this titration phase and doses higher than $300 \mathrm{mg}$ daily are often required to reach the target. ${ }^{1}$ Allopurinol therapy should not be stopped in the event of an acute gout flare and can be safely started during an acute attack. 16,17

Previously, based on studies published in the 1980s, renal function limited the maximum daily dose of allopurinol. ${ }^{18}$ However, basing the dose on creatinine clearance results in only $19 \%$ of patients reaching the target serum urate. ${ }^{8}$ The final dose of allopurinol needed to reach the target is predicted by the pretreatment urate concentration, not renal function. Higher doses are required in patients with higher pretreatment serum urate concentrations. ${ }^{19}$

In patients with renal impairment, allopurinol should be started at a low dose and escalated more slowly than in other patients to achieve the target urate concentration.' For example, patients with an estimated glomerular filtration rate less than $30 \mathrm{~mL} /$ minute may start at $50 \mathrm{mg}$ every second day. The maximum dose of allopurinol required to reach the target should be determined by tolerability, not renal function.

\section{Adverse effects}

Drinking plenty of liquids and eating little and often can help to reduce the most common adverse effects of nausea or vomiting. Less commonly, allopurinol can cause a rash or flaking of the skin. Allopurinol must be ceased and medical advice sought promptly if any rash develops, especially if the very rare adverse effects of mouth ulceration or a severe skin rash develop. Other adverse effects include altered taste, drowsiness and diarrhoea.

A rare but potentially fatal adverse event is allopurinol hypersensitivity syndrome. This is characterised by rashes (e.g. Stevens-Johnson syndrome, toxic epidermal necrolysis), eosinophilia, leucocytosis, fever, hepatitis and renal failure. The mortality is reported to be as high as $27 \% .^{20}$ The mechanisms leading to allopurinol hypersensitivity syndrome are unclear. Risk factors for its development include female sex, age, renal impairment, diuretic use and, in some ethnic groups, the HLA-B*5801 genotype. ${ }^{20}$ (People of Asian descent, especially the Han Chinese, have a higher frequency of the HLA-B*5801 allele. ${ }^{8}$ ) A higher starting dose and quick escalation are associated with an increased risk of developing allopurinol hypersensitivity syndrome. Approximately $90 \%$ of cases occur within the first three months of starting treatment. ${ }^{20}$ For patients who start allopurinol successfully, there is no association between the maintenance dose and allopurinol hypersensitivity syndrome. ${ }^{8}$ This supports the notion of a 'start low and go slow' approach to allopurinol dosing, especially in those with risk factors for hypersensitivity syndrome.

\section{Febuxostat}

Febuxostat is a new xanthine oxidase inhibitor but, unlike allopurinol, it is not a purine analogue. It has been effective in a number of trials and is approved in Australia for the treatment of gout in patients who are unable to tolerate allopurinol. Febuxostat is metabolised by the liver and renal excretion is not a major route of elimination. A dose of febuxostat $40 \mathrm{mg}$ per day is clinically equivalent to allopurinol $300 \mathrm{mg}$ in efficacy. If the serum uric acid is greater than $0.36 \mathrm{mmol} / \mathrm{L}$ after 2-4 weeks of therapy, febuxostat $80 \mathrm{mg}$ once daily is recommended.

Febuxostat has relatively few drug interactions. It may be safe to use in patients with renal impairment, ${ }^{2}$ however the efficacy and safety of febuxostat has not been fully evaluated in patients with a creatinine clearance less than $30 \mathrm{~mL} /$ minute. Also, there are some concerns about possible cardiovascular events associated with febuxostat and it costs more than allopurinol. ${ }^{2}$ Febuxostat is contraindicated in patients with ischaemic heart disease or congestive heart disease and, like allopurinol, is not recommended in patients taking azathioprine or mercaptopurine.

\section{Uricosuric drugs}

Uricosurics promote the renal excretion of uric acid and are effective for controlling serum urate. Drugs such as probenecid inhibit organic anion transporters (OATs) in the kidney, which are responsible for the reabsorption of filtered uric acid. ${ }^{8}$ Caution is required in those with a history of kidney stones because uricosurics can precipitate uric acid stones. ${ }^{8}$ In patients at risk of renal calculi, if no other option is available, increased fluid intake and urinary 


\section{SELF-TEST QUESTIONS}

True or false?

1. Allopurinol is not indicated for the treatment of asymptomatic hyperuricaemia.

2. Allopurinol should not be started during an acute attack of gout.

Answers on page 143 alkalinisation may be considered. Probenecid is effective in patients with impaired renal function, contrary to previously held beliefs. ${ }^{8}$

Benzbromarone is a potent uricosuric drug that is available in many countries but not Australia. It is effective as a sole drug in the treatment of gout. When used as add-on therapy in combination with allopurinol, more than $90 \%$ of patients reach a serum urate concentration of less than $0.30 \mathrm{mmol} / \mathrm{L}^{8}{ }^{8}$

Lesinurad is another uricosuric drug that is currently in clinical trials. It is an inhibitor of uric acid transporters in the renal tubule (urate anion exchanger 1 (URAT1) and organic anion transporter 4 (OAT4)). ${ }^{8}$

\section{Uricases}

Uricases (such as rasburicase, a recombinant urate oxidase) metabolise urate to a more soluble form which is then excreted in the urine. They are highly effective at reducing serum urate and treating patients with severe gout, ${ }^{21}$ however they are not approved in Australia for this indication. As uricases are proteins, allergic reactions such as rashes, urticaria and bronchospasm are potential complications, especially after repeated infusions. ${ }^{8,21}$

\section{Conclusion}

The burden of gout is growing worldwide, due to the increasing number of people with conditions that predispose them to hyperuricaemia such as hypertension, obesity, diabetes, chronic kidney disease and the use of diuretics. ${ }^{14}$ Urate-lowering therapy reduces the risk of further attacks of gout, but prophylaxis against flares is required until the maintenance dose is stabilised. $<$

\section{Conflicts of interest: Andrew Finch attended Editorial Executive Committee meetings as the clinical} pharmacology registrar for Australian Prescriber in 2015.

Conflicts of interest: Paul Kubler was site investigator in multinational Phase III gout studies evaluating lesinurad (Ardea Biosciences, now taken over by AstraZenica). Patient payments went into a public hospital research trust fund (no personal benefit).

\section{REFERENCES}

1. Graf SW, Whittle SL, Wechalekar MD, Moi JH, Barrett C, Hill CL, et al. Australian and New Zealand recommendations for the diagnosis and management of gout: integrating systematic literature review and expert opinion in the 3e Initiative. Int J Rheum Dis 2015;18:341-51. http://dx.doi.org/ 10.1111/1756-185X.12557

2. Robinson PC, Horsburgh S. Gout: joints and beyond, epidemiology, clinical features, treatment and co-morbidities. Maturitas 2014;78:245-51. http://dx.doi.org/10.1016/j.maturitas.2014.05.001

3. Zhu Y, Pandya BJ, Choi HK. Comorbidities of gout and hyperuricemia in the US general population: NHANES 2007-2008. Am J Med 2012;125:679-687.e1. http://dx.doi.org/10.1016/j.amjmed.2011.09.033

4. Robinson PC, Taylor WJ, Merriman TR. Systematic review of the prevalence of gout and hyperuricaemia in Australia. Intern Med J 2012;42:997-1007.

5. Smith EU, Díaz-Torné C, Perez-Ruiz F, March LM. Epidemiology of gout: an update. Best Pract Res Clin Rheumatol 2010;24:811-27. http://dx.doi.org/ 10.1016/j.berh.2010.10.004

6. Galassi FM, Borghi C. A brief history of uric acid: from gout to cardiovascular risk factor. Eur J Intern Med 2015;26:373. http://dx.doi.org/ 10.1016/j.ejim.2015.04.005

7. Perez-Ruiz F, Dalbeth N, Bardin T. A review of uric acid, crystal deposition disease, and gout. Adv Ther 2015;32:31-41. http://dx.doi.org/10.1007/ s12325-014-0175-z

8. Robinson PC, Dalbeth N. Advances in pharmacotherapy for the treatment of gout. Expert Opin Pharmacother 2015;16:533-46. http://dx.doi.org/10.1517/ 14656566.2015.997213

9. Australian medicines handbook. Adelaide: Australian Medicines Handbook Pty Ltd; 2016. https://shop.amh.net.au/products/books/2016 [cited 2016 Jul 1]

10. Finch A, Pillans PI. P-glycoprotein and its role in drug-drug interactions. Aust Prescr 2014;37:137-9. http://dx.doi.org/10.18773/austprescr.2014.050

11. Janssens $\mathrm{HJ}$, Janssen $\mathrm{M}$, van de Lisdonk EH, van Riel PL, van Weel C. Use of oral prednisolone or naproxen for the treatment of gout arthritis: a doubleblind, randomised equivalence trial. Lancet 2008;371:1854-60. http://dx.doi.org/10.1016/S0140-6736(08)60799-0

12. eTG complete [Internet]. Melbourne: Therapeutic Guidelines Limited; 2016. www.tg.org.au [cited $2016 \mathrm{Jul} 1]$
13. Khanna D, Fitzgerald JD, Khanna PP, Bae S, Singh MK, Neogi T, et al.; American College of Rheumatology. 2012 American College of Rheumatology guidelines for management of gout. Part 1: systematic nonpharmacologic and pharmacologic therapeutic approaches to hyperuricemia. Arthritis Care Res (Hoboken) 2012;64:1431-46. http://dx.doi.org/10.1002/acr.21772

14. Lippi G, Mattiuzzi C, Cervellin G. Meat consumption and gout: friend, foe or neither? Rheumatol Int 2015:35:1443-4. http://dx.doi.org/10.1007/ s00296-015-3254-9

15. Choi HK. A prescription for lifestyle change in patients with hyperuricemia and gout. Curr Opin Rheumatol 2010;22:165-72. http://dx.doi.org/10.1097/ BOR.0b013e328335ef38

16. Taylor TH, Mecchella JN, Larson RJ, Kerin KD, Mackenzie TA Initiation of allopurinol at first medical contact for acute attacks of gout: a randomized clinical trial. Am J Med 2012;125:1126-1134.e7. http://dx.doi.org/10.1016/ j.amjmed.2012.05.025

17. Turner J, Cooper D. Does colchicine improve pain in an acute gout flare? Ann Emerg Med 2015;66:260-1. http://dx.doi.org/10.1016/ j.annemergmed.2015.04.006

18. Hande KR, Noone RM, Stone WJ. Severe allopurinol toxicity. Description and guidelines for prevention in patients with renal insufficiency. Am J Med 1984;76:47-56. http://dx.doi.org/10.1016/0002-9343(84)90743-5

19. Stamp LK, Taylor WJ, Jones PB, Dockerty JL, Drake J, Frampton C, et al. Starting dose is a risk factor for allopurinol hypersensitivity syndrome: a proposed safe starting dose of allopurinol. Arthritis Rheum 2012;64:2529-36. http://dx.doi.org/10.1002/art.34488

20. Graham GG, Kannangara DR, Stocker SL, Portek I, Pile KD, Indraratna PL, et al. Understanding the dose-response relationship of allopurinol: predicting the optimal dosage. Br J Clin Pharmacol 2013;76:932-8. http://dx.doi.org/10.1111/ bcp.12126

21. Becker MA, Baraf HS, Yood RA, Dillon A, Vázquez-Mellado J, Ottery FD, et al. Long-term safety of pegloticase in chronic gout refractory to conventional treatment. Ann Rheum Dis 2013;72:1469-74. http://dx.doi.org/10.1136/ annrheumdis-2012-201795

\section{FURTHER READING}

For consumer information regarding both gout and the drugs used to treat gout, visit the Arthritis Australia website: www.arthritisaustralia.com.au 